\title{
Tau-Mediated Cytotoxicity in a Pseudohyperphosphorylation Model of Alzheimer's Disease
}

\author{
Thomas Fath, ${ }^{1,2}$ Jochen Eidenmüller, ${ }^{2}$ and Roland Brandt, ${ }^{1,2}$ \\ 1Department of Neurobiology, University of Osnabrück, 49076 Osnabrück, Germany, and 2Interdisziplinäres Zentrum für \\ Neurowissenschaften, Department of Neurobiology, University of Heidelberg, 69120 Heidelberg, Germany
}

Aggregation and increased phosphorylation of tau at selected sites ("hyperphosphorylation") are histopathological hallmarks of Alzheimer's disease (AD). However, it is not known whether the tau pathology has a primary role during neuronal degeneration. To determine the role of tau hyperphosphorylation in AD, pseudohyperphosphorylated tau (PHP-tau) that simulates disease-like permanent, high stoichiometric tau phosphorylation and mimics structural and functional aspects of hyperphosphorylated tau was expressed in neural cells. In differentiated PC12 cells, PHP-tau exhibited reduced microtubule interaction and failed to stabilize the microtubule network compared with exogenously expressed wild-type tau (wt-tau). During longer culture, PHP-tau exerted a cytotoxic effect, whereas wt-tau was neutral. PHP-tau-mediated cytotoxicity was associated with an induction of apoptotic cell death as characterized by chromatin condensation, DNA fragmentation, and

Abnormal tau processing and extensive neuron loss are neuropathological hallmarks of a family of heterogeneous neurodegenerative diseases collectively known as tauopathies (Lee et al., 2001). An example is Alzheimer's disease (AD), which is characterized by intracellular aggregates consisting of hyperphosphorylated tau protein in paired helical filaments (PHFs) and extracellular amyloid plaques composed of $\mathrm{A} \beta$. Abnormal phosphorylation of tau is considered one of the earliest signs of neuronal degeneration and appears to precede tau aggregation or amyloid formation (Braak et al., 1994). However, it is not known whether tau hyperphosphorylation has a primary role during neuronal degeneration and which intracellular mechanisms are involved.

Hyperphosphorylated tau isolated from patients with AD exhibits a conformational change, an increased immunoreactivity with antibodies that detect phosphorylated epitopes in selected regions of the protein, and a functional loss to promote microtubule assembly (Lu and Wood, 1993; Alonso et al., 1994). The transformation of tau into a hyperphosphorylated state appears to involve a concerted and sequential action of several kinases and phosphatases (Matsuo et al., 1994; Jicha et al., 1999b; Patrick et

\footnotetext{
Received May 7, 2002; revised July 31, 2002; accepted Aug. 16, 2002.

This work was supported by the Deutsche Forschungsgemeinschaft Schwerpunkt Zelluläre Mechanismen der Alzheimer Erkrankung (BR 1192/10-1,2) (R.B.), the Alzheimer Forschungsinitiative (R.B.), and a fellowship of the graduate college Molecular and Cellular Neurobiology (T.F.). We thank A. Hellwig for electron microscopy and Dr. Neelam Shahani for critically reading this manuscript.

Correspondence should be addressed to Dr. Roland Brandt, Department of Neurobiology, University of Osnabrück, Barbarastraße 11, D-49076 Osnabrück, Germany. E-mail: Brandt@biologie.uni-osnabrueck.de.

Copyright (C) 2002 Society for Neuroscience $0270-6474 / 02 / 229733-09 \$ 15.00 / 0$
}

caspase- 3 activation in the absence of detectable protein aggregates. Furthermore, PHP-tau expression specifically sensitized the cells for other apoptotic stimuli (colchicine and staurosporine). Herpes simplex virus-mediated overexpression of PHP-tau induced degeneration associated with an induction of apoptotic mechanisms also in terminally differentiated human CNS model neurons. Partially pseudophosphorylated constructs caused an intermediate toxicity. The data provide evidence for a neurotoxic "gain of function" of soluble tau during $A D$ as a result of structural changes that are induced by a cumulative, high stoichiometric tau phosphorylation. PHP-tauexpressing cells and organisms could provide a useful system to identify mechanisms that contribute to tau-mediated toxicity.

Key words: tau; Alzheimer's disease; phosphorylation; apoptosis; hyperphosphorylation; human model neurons

al., 1999). Although few if any phosphorylation sites are unique to tau in PHFs (PHF-tau), the proportion of tau phosphorylation at any given site is significantly higher in PHF-tau than in biopsyderived normal tau protein (Matsuo et al., 1994; MorishimaKawashima et al., 1995). Thus, the phosphorylation abnormality in PHF-tau appears to be an increase in the stoichiometry of phosphate incorporated at selected sites.

We have shown previously that mutated tau proteins in which serine/threonine residues (which are phosphorylated to a high extent in PHF-tau) were substituted with glutamate to create a pseudophosphorylation of these residues (PHP-tau) (see Fig. 1A) mimic key structural and functional aspects of hyperphosphorylated tau protein (Eidenmüller et al., 2000; Maas et al., 2000). In particular, recombinant PHP-tau exhibits a decreased $\beta$-sheet and an increased $\beta$-turn content and displays a functional deficit to promote microtubule assembly in vitro, all of which features are characteristic for hyperphosphorylated tau protein (Eidenmüller et al., 2000). In addition, PHP-tau was deficient to interact with the dominant brain phosphatase $2 \mathrm{~A}$ isoform $\mathrm{AB} \alpha \mathrm{C}$, no longer interacted with plasma membrane components, and exhibited a reduced aggregation into disease-like filaments (Eidenmüller et al., 2001). Thus, the use of PHP-tau could provide a useful tool to analyze the consequences of a disease-like permanent and high stoichiometric phosphorylation state of tau protein in a neuronal context.

In this study, we expressed wild-type tau (wt-tau) and pseudophosphorylated tau constructs in differentiated PC12 cells and terminally differentiated, postmitotic human CNS model neurons to examine effects of a disease-like tau modification in neural cells. The cells were analyzed for the distribution of tau proteins, 
induction of cell death, and signs of neurodegeneration, which correlated with structural changes in tau protein. The results provide evidence for a toxic "gain of function" of disease-like modified tau, which was independent of aggregation of tau. The data suggest that PHP-tau-expressing cells provide a useful model for analyzing aspects of tau pathology suitable for the development of high-throughput screening strategies.

\section{MATERIALS AND METHODS}

Materials. Chemicals were purchased from Sigma (Deisenhofen, Germany); cell culture media and supplements were from Invitrogen (Gaithersburg, MD); and culture flasks and dishes were from Nunc (Roskilde, Denmark), unless stated otherwise. Collagen was prepared from rat tails by acetic acid extraction.

Construction of expression vectors. Eukaryotic expression plasmids for fetal human tau (352 residues) with a fused FLAG epitope, to allow identification of the expressed proteins, were constructed in $\mathrm{pRc} / \mathrm{cyto}$ megalovirus (Invitrogen). PHP-tau was constructed by changing the codons for S198, S199, S202, T231, S235, S396, S404, S409, S413, and S422 to glutamate as described previously (Eidenmüller et al., 2000). As a control, an expression vector was constructed in which the same codons were changed to alanine (Ala-tau). In addition, two other expression plasmids for fetal human tau were constructed in which five residues in the proline-rich region $\left(\mathrm{PP}_{(\mathrm{P})}\right.$-tau $)$ or the $\mathrm{C}$-terminal region $\left.\left(\mathrm{PP}_{(\mathrm{C})}\right)^{-\mathrm{tau}}\right)$ were changed to glutamate. Viral vectors were produced by subcloning FLAG-epitope tagged wt-tau, $\mathrm{PP}_{(\mathrm{P})}$-tau, $\mathrm{PP}_{(\mathrm{C})}$-tau, and PHP-tau sequences encoding the fetal-specific 352 residues tau isoform or FLAGepitope tagged wt-tau and PHP-tau sequences encoding the adultspecific 441 residues tau isoform into herpes simplex virus (HSV)-1 expression vectors as described previously (Fath et al., 2000).

PC12 cell culture and transfection. PC12 cells were cultured in serumcontaining DMEM and transfected using Lipofectin as described previously (Brandt et al., 1995). Individual clones were picked and propagated in serum-DMEM supplemented with $250 \mu \mathrm{g} / \mathrm{ml}$ Geneticin on collagencoated culture dishes. For each construct, several independent clonal lines were selected that expressed comparable levels of the protein $\left(\sim 10^{7}\right.$ molecules of tau per cell as judged by immunoblot analysis of cellular lysates). For differentiation, cells were seeded at $10^{5}$ cells $/ \mathrm{cm}^{2}$ on collagen-coated surfaces in serum-free medium optimized for long-term neural culture [NB/B27 (Brewer et al., 1993)] in the presence of 100 $\mathrm{ng} / \mathrm{ml}$ nerve growth factor (NGF) (7S mNGF; Alomone Labs, Jerusalem, Israel).

Tau-microtubule binding assay. PC12 cells $\left(10^{6}\right)$ were differentiated for $2 \mathrm{~d}$, scraped off the plate, collected by centrifugation, resuspended in 20 $\mu \mathrm{l}$ of prewarmed extraction buffer $[0.1 \%(\mathrm{v} / \mathrm{v})$ Triton X-100, $0.1 \mathrm{M}$ 1,4-piperazinediethanesulfonic acid/ $\mathrm{KOH}, 1 \mathrm{~mm} \mathrm{MgSO}_{4}, 2 \mathrm{~mm}$ EGTA, $0.1 \mathrm{~mm}$ EDTA, and $2 \mathrm{M}$ glycerol, $\mathrm{pH}$ 6.75] containing protease inhibitors (1 mM PMSF, $10 \mu \mathrm{g} / \mathrm{ml}$ each of leupeptin and pepstatin, and $1 \mathrm{~mm}$ EGTA), and incubated for $8 \mathrm{~min}$ at $37^{\circ} \mathrm{C}$, and the pellet and supernatant fraction was separated by centrifugation $\left(15 \mathrm{~min}\right.$ at $15,000 \times g$ and $\left.25^{\circ} \mathrm{C}\right)$. Equal amounts of pellet and supernatant fraction were separated by SDS-PAGE, blotted, and processed for immunodetection with anti-tau and anti-tubulin antibodies.

Culture of NT2-N neurons and infection with HSV-1. Terminally differentiated NT2-N cells were produced by in vitro differentiation of NT2 cells for 5 weeks, enriched by serial replatement, and treated with cytostatica as described previously (Piontek et al., 1999). For all experiments, cells were used 1-2 weeks after cytostatica treatment. At this stage, the neurons were postmitotic and exhibited a polar cytoarchitecture as judged by an axon-specific tau staining. NT2-N neurons were seeded at 2500 or 5000 cells per squared centimeter on Matrigel (Becton Dickinson)-coated coverslips in serum-DMEM or seeded at 7500 cells per squared centimeter on Matrigel in four-well plates. Amplification and purification of HSV-1 constructs were performed as described previously (Fath et al., 2000). For all virus preparations, the ratio of amplicon to helper was close to 1:1 as determined by immunofluorescence microscopy. For immunocytochemistry, cells were cultured for $3 \mathrm{~d}$ and infected with virus to yield $\sim 50 \%$ tau-expressing neurons. For immunoblot analysis, $7.5 \times 10^{3}$ NT2-N neurons were seeded in Matrigelcoated four-well plates and infected after $3 \mathrm{~d}$ with virus.

Immunocytochemistry and immunoblot analysis. Cells were fixed with $4 \%$ paraformaldehyde followed by permeabilization with Triton X-100 or using a combined NP-40-extraction-fixation protocol (Brandt et al.,
1995). Staining used monoclonal mouse antibody against the FLAGepitope (M5), monoclonal anti-rat antibody against tubulin (YL1/2) (Kilmartin et al., 1982), Cy3-coupled anti-mouse, and fluorescein isothiocyanate-coupled anti-rat antibody (secondary antibodies from Dianova). To stain nuclei, $5 \mu \mathrm{g} / \mathrm{ml} \mathrm{4,6-diamidino-2-phenylindole} \mathrm{(DAPI)}$ was included in the secondary antibody mixture. Cells were photographed using Neofluar lenses on a Zeiss Axioskop and a Leica TCS 4D laser-scanning microscope.

For immunoblot analysis, cells were lysed in RIPA buffer $(50 \mathrm{~mm}$ Tris/HCl, pH 7.5, 150 mm NaCl, 1 mm EDTA, 1\% NP-40, 0.5\% deoxycholate, and $0.1 \%$ SDS) containing protease inhibitors (1 mM PMSF, 10 $\mu \mathrm{g} / \mathrm{ml}$ each of leupeptin and pepstatin, 1 mM EGTA) and phosphatase inhibitors $(1 \mathrm{~mm}$ sodium orthovanadate, $20 \mathrm{~mm} \mathrm{NaF}$, and $1 \mathrm{~mm}$ sodium pyrophosphate), incubated for $30 \mathrm{~min}$ at $4^{\circ} \mathrm{C}$, and centrifuged for $10 \mathrm{~min}$ at $13,000 \times g$, and the supernatant (lysate) was collected. After electrophoretic separation, gels were blotted and stained with antibodies against tau (Tau5; PharMingen, San Diego, CA), actin (Amersham Biosciences), $\alpha$-tubulin (DM1A), and acetylated tubulin (6-11-B1). As secondary antibody, horseradish peroxidase-coupled goat anti-mouse antiserum (Jackson ImmunoResearch, West Grove, PA) was used. Detection used enhanced chemiluminescence (Amersham Biosciences) and was performed according to the manufacturer's protocol. Quantification of the blots was performed with a flatbed scanner (Agfa Studioscan IIsi) and the program NIH Image 1.61/ppc.

Electron microscopy. PC12 cells were scraped off the dish, collected by centrifugation, fixed with $2 \%$ glutaraldehyde in $0.1 \mathrm{~m}$ sodium cacodylate, $\mathrm{pH} 7.4$, and processed for ultrastructural analysis as described previously (Brandt et al., 1995). Electron microscopy was performed on a Zeiss 10CR electron microscope at $60 \mathrm{kV}$.

Other methods. Cell toxicity was assessed by trypan blue exclusion of resuspended PC12 cells and by 3-(4,5-dimethylthiazol-2-yl)-2,5diphenyltetrazolium bromide (MTT) conversion assays. For MTT conversion assays, PC12 cells were cultured in triplicate in 96-well plates. MTT conversion was determined as described previously (Piontek et al., 1999). When indicated, colchicine or staurosporine was added from a 100 $\mathrm{mg} / \mathrm{ml}$ stock in ethanol (colchicine) or a $1 \mathrm{~mm}$ stock in dimethylsulfoxide (staurosporine).

Terminal deoxynucleotidyl transferase nick end-labeling (TUNEL) was performed using a commercial fluorescein apoptosis detection system (Promega, Madison, WI) according to the manufacturer's protocol and analyzed by visual inspection with $40 \times$ Neofluar lenses on a Zeiss Axiophot.

For determination of caspase- 3 activity, cells were scraped off the dish, collected by centrifugation, distributed in aliquots containing $10^{6}$ cells, and assayed using a colorimetric kit (Clontech, Cambridge, UK) according to the manufacturer's protocol. Caspase-3 activity was calculated from the difference without and with the caspase-3 inhibitor Asp-GluVal-fluoromethyl ketone and a standard curve established with the chromogenic product $p$-nitroanilide.

Comparison between experimental groups was based on paired Student's $t$ test $\left({ }^{*} p<0.05 ;{ }^{* *} p<0.01 ;{ }^{* *} p<0.001\right)$.

\section{RESULTS}

\section{Simulation of a disease-like tau hyperphosphorylation in cells}

The phosphorylation abnormality in tau from patients with AD appears to be an increased stoichiometry and a decreased turnover of phosphate incorporated at selected sites. To analyze functional consequences of a disease-like state of tau protein in a neuronal context, wt-tau and PHP-tau (Fig. $1 A$ ), which mimic key structural and functional aspects of hyperphosphorylated tau protein (Eidenmüller et al., 2000; Maas et al., 2000), were expressed in rat pheochromocytoma (PC12) cells. Stable PC12 clones that constitutively express human fetal wt-tau and PHPtau were produced. The constructs were epitope tagged with the FLAG sequence at the $\mathrm{N}$ terminus to distinguish them from endogenously expressed tau protein isoforms (Smith et al., 1995). As a control, cell lines with the vector containing the FLAG but lacking tau sequence (vector control) were constructed. Individual cell lines were chosen that expressed wt-tau and PHP-tau at a comparable level as judged by immunocytochemistry with an 
A

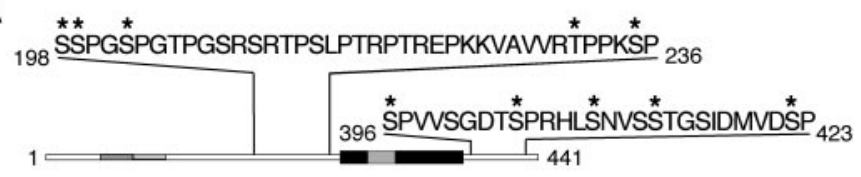

B

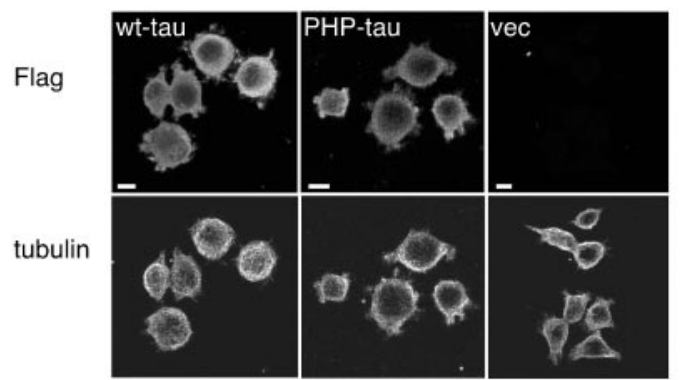

C

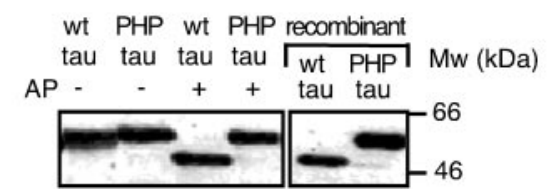

Figure 1. Simulation of a disease-like tau hyperphosphorylation in cells. $A$, Residues that constitute sites with an increased phosphorylation in PHF-tau (Morishima-Kawashima et al., 1995) and have been substituted for glutamate to simulate a disease-like hyperphosphorylation (PHP-tau) are indicated by asterisks. The microtubule-binding repeats of tau are indicated by the thick black box. Adult-specific exons are shaded. B, Fluorescence staining for exogenous tau expression in undifferentiated PC12 cells stably transfected with wt-tau, PHP-tau, or a vector control (vec). $C$, Immunoblots of cellular lysates from undifferentiated PC12 cells stably transfected with wt-tau or PHP-tau. Lysates remained untreated (left) or were preincubated with alkaline phosphatase $(A P)$ to remove phosphate residues (middle). For comparison, recombinant wt-tau and PHP-tau are shown on the right. B, Cells were plated at $2 \times 10^{4}$ cells per squared centimeter on collagen-coated coverslips, fixed on the next day with $4 \%$ paraformaldehyde, and processed for immunofluorescence, as described in Materials and Methods, using a mouse monoclonal antibody against the FLAG-epitope (M5, top) and a rat monoclonal antibody against tubulin (YL1/2, bottom). $C$, Lysates corresponding to $2.5 \times 10^{5}$ cells were loaded, separated by SDS-PAGE on $10 \%$ acrylamide, and detected with anti-tau (Tau5) antibody. To remove covalently bound phosphates, lysates were treated with $5 \mathrm{U}$ of alkaline phosphatase overnight at $37^{\circ} \mathrm{C}$. Scale bars, $10 \mu \mathrm{m}$.

anti-FLAG antibody (Fig. $1 B$ ). Immunoblots of cellular lysates confirmed that the cell populations expressed tau to a similar level, which corresponded to an average of $\sim 10^{7}$ molecules of tau per cell (Fig. 1C). At the conditions used, no endogenous tau was detected by immunoblot with anti-tau antibody (Tau5) (data not shown) because of the high expression level of exogenous tau and the fact that the antibody reacts better with human than with rat tau sequence (Maas et al., 2000). In cellular lysates, wt-tau and PHP-tau both separated as single bands (Fig. 1C, left). Phosphatase treatment increased the mobility of wt-tau but not of PHPtau to the state observed for the recombinant proteins (Fig. $1 C$, right), indicating extensive phosphorylation of wt-tau in the mitotic cells that changes the conformation of wt-tau similar to that of PHP-tau. In growing cultures, no effect of the expressed protein on the survival of the cells or on the generation time was observed compared with a control line that was transfected with the vector construct lacking tau sequence (data not shown).

To determine the effect of wt-tau and PHP-tau in differentiated cells, the respective lines were treated with NGF in chemically defined medium (NB/B27). Under these conditions, the cells
A

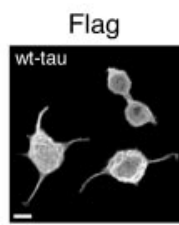

tubulin

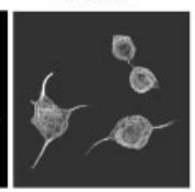

Flag
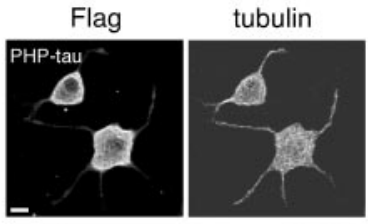

B

Flag
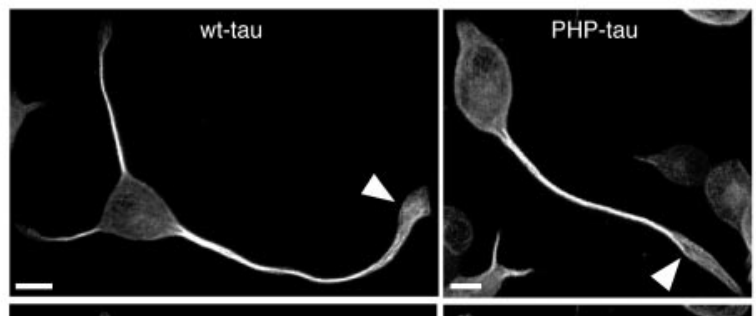

tubulin
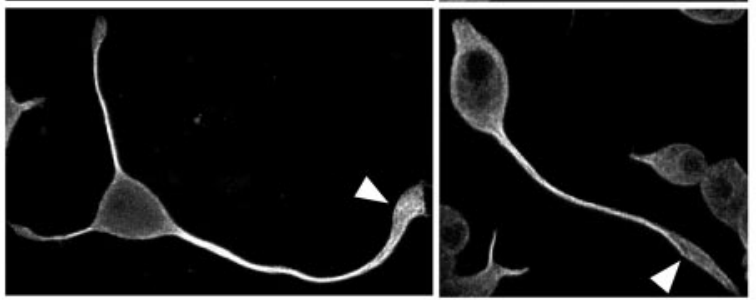

C
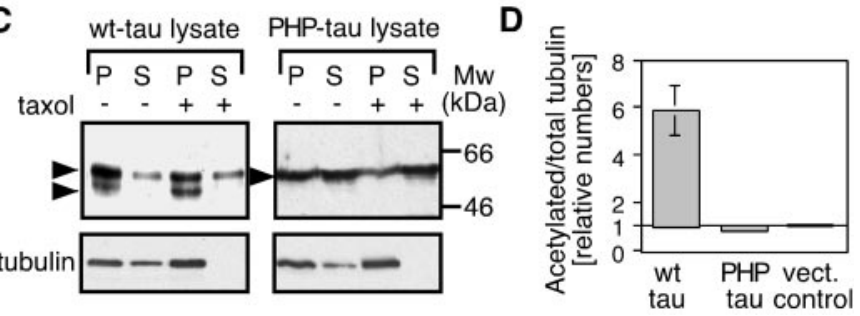

Figure 2. Expression and microtubule association of wt-tau and PHP-tau in differentiated PC12 cells. $A$, Fluorescence staining for exogenous tau expression in differentiated PC12 cells stably transfected with wt-tau and PHP-tau. B, Fluorescence staining for exogenous tau and cellular microtubules of differentiated wt-tau- and PHP-tau-expressing PC12 cells after a combined extraction-fixation protocol to demonstrate cytoskeletal association. Note the colocalization of tau and microtubules that is evident in growth cone-like structures (arrowheads). $C$, Immunoblot of cytoskeletally associated pellet $(P)$ and nonassociated supernatant $(S)$ fraction after fractionation of lysates from differentiated PC12 cells. Populations of tau phosphoisoforms with fast and slow electrophoretic mobilities are indicated. Fractionations were also performed in the presence of $10 \mu \mathrm{M}$ taxol to completely polymerize the microtubule network. $D$, Ratio of acetylated/total tubulin as determined by immunoblot analysis of cellular lysates from differentiated PC12 cells. Numbers are normalized to the ratio of acetylated/total tubulin in control cells (set as 1). For immunofluorescence analysis, cells were differentiated for $4 \mathrm{~d}$ with NGF, fixed using $4 \%$ paraformaldehyde $(A)$ or a combined NP-40 extraction-fixation protocol $(B)$, and processed for immunofluorescence using anti-FLAG (M5) and anti-tubulin (YL1/2) antibody as described in Materials and Methods. Scale bars, $10 \mu \mathrm{m}$. For immunoblots, lysates corresponding to 0.5-1.5 $\times 10^{6}$ cells were loaded and detected with anti-tau (Tau5), anti-tubulin (DM1A), or anti-acetylated tubulin (6-11B-1) antibody. Quantifications were from four experiments using two independent clonal lines per construct. Mean and SE are shown.

cease to proliferate, upregulate neuronal marker proteins, and establish neurites (Greene et al., 1991). After induction of differentiation, the cells continued to express the tau constructs at a similar level (Fig. 2A). To analyze the interaction of tau with the microtubule skeleton, cells were processed using a combined extraction-fixation protocol and analyzed by laser-scanning microscopy. Both constructs showed a filamentous staining pattern, which colocalized with the distribution of microtubules in pro- 
cesses and growth cone-like structures (Fig. $2 B$ ), indicating that the expressed proteins were capable of interacting with the microtubule skeleton. To quantitate the extent of binding of tau to microtubules, cellular lysates were fractionated into cytoskeletally associated and nonassociated fractions. Wt-tau protein was present almost exclusively in the cytoskeleton fraction (Fig. 2C). In contrast, the majority of PHP-tau was present in the supernatant, indicating that PHP-tau interacts less with the neural cytoskeleton. Treatment with taxol, which leads to a complete polymerization of tubulin, did not affect the distribution of wt-tau or PHP-tau, indicating that the different distribution was a property of the respective tau construct rather than a consequence of the assembly state of the microtubule skeleton. To determine a potential effect of the constructs on the stability of the microtubule network, the ratio of acetylated microtubules known to serve as a marker for stable microtubule subpopulations (Piperno et al., 1987) to total microtubule polymer was determined by quantitative immunoblotting. The relative amount of acetylated microtubules was approximately fivefold increased in cells expressing wt-tau protein compared with vector control cells (Fig. 2D). In contrast, no effect of the expression of PHP-tau was observed, indicating that the presence of PHP-tau is neutral with respect to the assembly state of the cellular microtubules. Taken together, the data indicate that PHP-tau shows a reduced interaction with microtubules and does not stabilize the microtubule network in cells as would be expected for tau species with an increased phosphorylation. No evidence for a direct effect of PHP-tau to induce microtubule destabilization was obtained.

\section{Pseudohyperphosphorylated tau exerts cytotoxicity in differentiated PC12 cells}

To determine a potential cytotoxic effect of tau expression in differentiated neural cells, wt-tau- or PHP-tau-expressing cells were differentiated with NGF at chemically defined conditions and processed for immunofluorescence after 3 and $7 \mathrm{~d}$ in culture. Under these conditions, most of the wt-tau-transfected cells survived and continued expressing tau protein (Fig. $3 A$, top). In contrast, although many PHP-tau-expressing cells were still present after $3 \mathrm{~d}$ in culture, most of the cells appeared degenerated after 1 week, as judged by the absence of PHP-tau staining and presence of condensed nuclei with a strong immunofluorescence for the nuclear dye DAPI (Fig. $3 A$, bottom). To quantify the survival of the cells, culture conditions were established in which $\sim 50 \%$ of the initially plated control cells survived as judged by Trypan Blue exclusion. Under this condition, less than half as many PHP-tau-expressing cells survived compared with vectortransfected control cells or cells that express wt-tau (Fig. 3B). As an independent measure, a bioassay (MTT assay) that detects mitochondrial activity reflecting the number of living cells gave very similar results (Fig. 3C). The data indicate that PHP-tau exerts a cytotoxic effect in differentiated PC12 cells, whereas wt-tau was neutral in both assays.

To determine morphological alterations of cells as a result of the expression of wt-tau or PHP-tau, the ultrastructural appearance of cells was analyzed. Many PHP-tau-expressing cells (40\% compared with 14 and $6 \%$ of wt-tau-expressing and control cells, respectively; 50 cells were evaluated for each PC12 line) showed multiple large aggregates of condensed chromatin in the nuclei (Fig. 4), representing a late event in degenerating cells. We did not observe obvious intracellular aggregates or filamentous structures that may represent aggregated tau in any of the cells
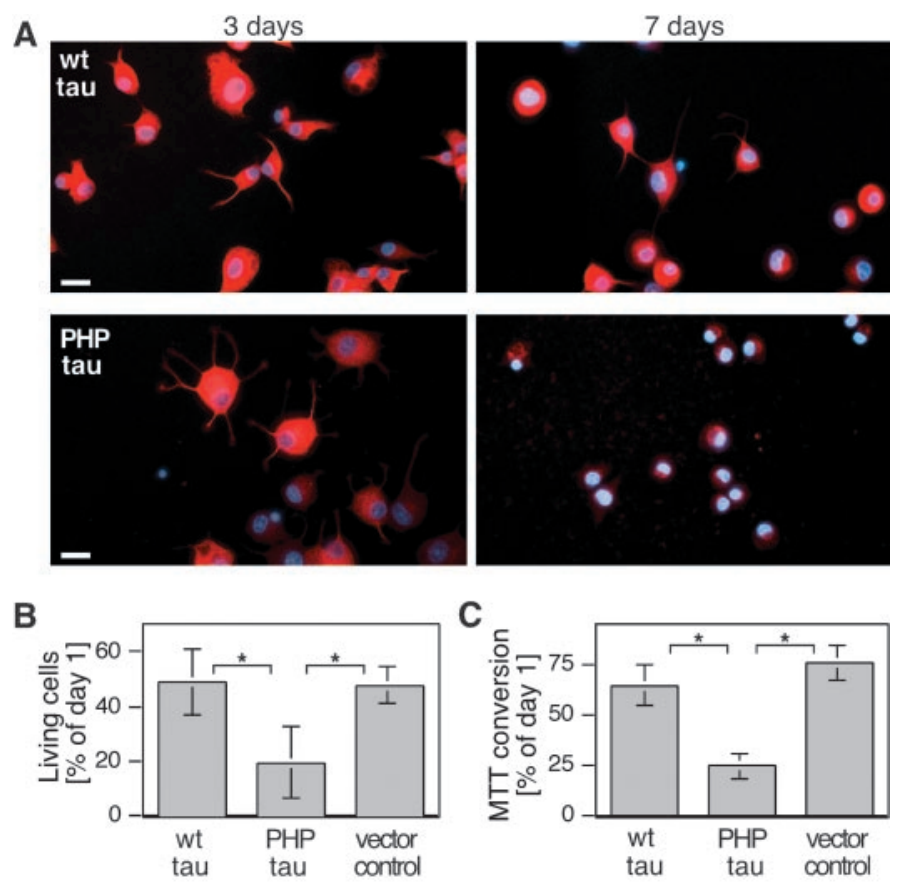

Figure 3. Reduced survival of differentiated PC12 cells stably transfected with PHP-tau. $A$, Fluorescence staining for exogenous tau (red) and nuclei (blue) of wt-tau-expressing (top) and PHP-tau-expressing (bottom) $\mathrm{PC} 12$ cells 3 and $7 \mathrm{~d}$ after induction of differentiation. $B, C$, Number $(B)$ and MTT conversion $(C)$ of differentiated $\mathrm{PC} 12$ cells. Survival was significantly reduced in PHP-tau-expressing cells. Cultures were for 1 week $(B$, $C$ ) or indicated times $(A)$ after the induction of differentiation with NGF. Cells were fixed with $4 \%$ paraformaldehyde and stained against the FLAG-epitope tag (M5) and with DAPI. Quantifications were from six experiments using two independent clonal lines per construct. Mean and SE are shown. Scale bars, $20 \mu \mathrm{m}$.
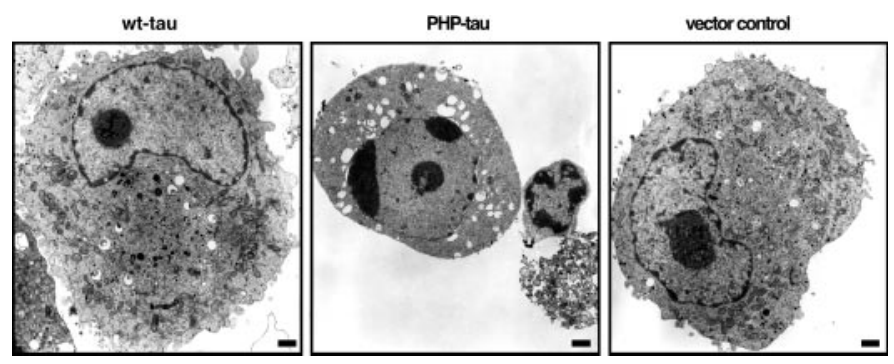

Figure 4. Ultrastructural changes in PC12 cells expressing PHP-tau. Compared with wt-tau-expressing and vector control cells, PHP-tauexpressing cells showed chromatin condensation. No evidence for abnormal protein aggregates was observed in any of the lines analyzed. Cells were cultured in serum-free medium for 1 week after the induction of differentiation with NGF and processed for electron microscopy as described in Materials and Methods. Scale bars, $1 \mu \mathrm{m}$.

evaluated, suggesting that the observed cytotoxicity is caused by soluble PHP-tau rather than formation of tau aggregates.

\section{The cytotoxicity of PHP-tau is associated with an induction of apoptotic mechanisms}

To determine the mechanisms that are involved in the cytotoxicity of PHP-tau, TUNEL assays, which detect DNA fragmentation characteristic of apoptosis, were performed. More than $70 \%$ of PHP-tau-expressing cells but only $30-40 \%$ of cells expressing wt-tau or control cells were TUNEL positive, suggesting that apoptotic mechanisms contribute to PHP-tau-mediated cell death 
A
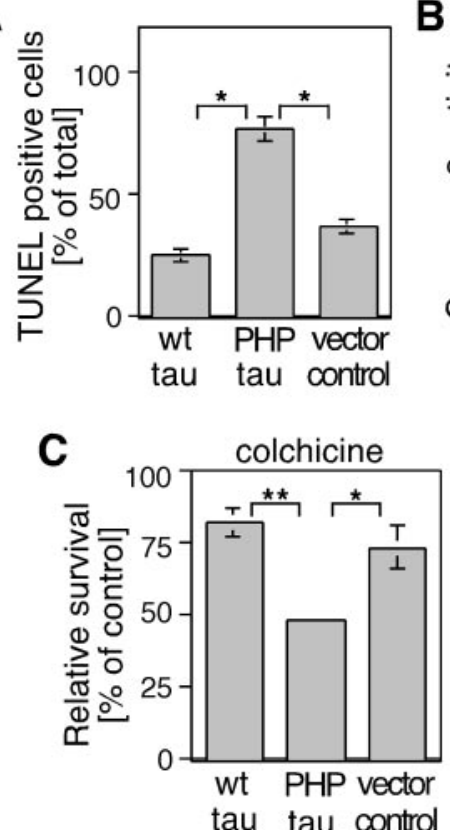

B
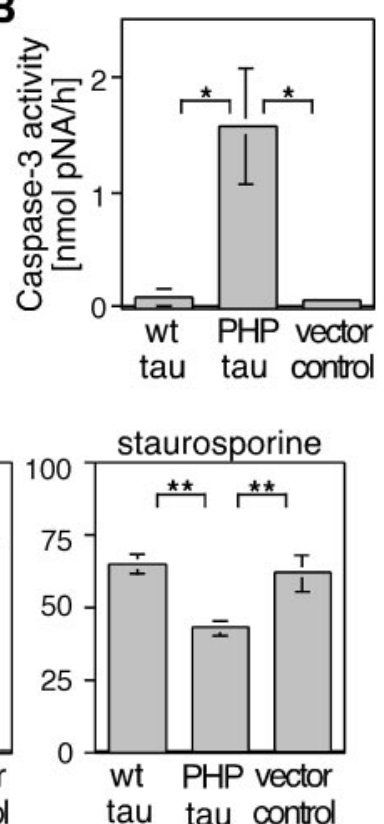

Figure 5. Induction of apoptosis in PHP-tau-expressing PC12 cells. $A, B$, Quantification of TUNEL-positive cells $(A)$ and caspase-3 activity $(B)$ in cell lines expressing wt-tau, PHP-tau, or a control line. The ratio of TUNEL-positive cells/level of caspase-3 activity is increased in PHP-tauexpressing cells. $C$, Effect of colchicine (left) and staurosporine (right) on the relative survival of stably transfected PC12 lines as analyzed by MTT conversion. PHP-tau-expressing cells show a reduced relative survival in the presence of both drugs compared with wt-tau-expressing cells or control lines. Cells were cultured in serum-free medium for $5 \mathrm{~d}(A, B)$ or 1 week $(C)$ after the induction of differentiation with NGF. $A$, The ratio of TUNEL-positive cells/total cells was determined by visual inspection from 10 microscopic frames. An independent experiment gave very similar results. $B, C$, Four experiments with two independent clonal lines were evaluated. Mean and SE are shown. $C$, Relative survival of cells treated with colchicine or staurosprine $(0.1 \mu \mathrm{g} / \mathrm{ml}$ and $100 \mathrm{nM}$, respectively; added twice, after 4 and 6 d) was measured against the survival of cultures in the absence of drugs.

(Fig. 5A). It is known that apoptosis is associated with an activation of the caspase cascade (Thornberry and Lazebnik, 1998). To investigate a potential induction of this cascade, activation of an effector caspase (caspase-3) was analyzed in a colorimetric assay. In PHP-tau-expressing cells, a significant increase in caspase-3 activity was observed compared with wt-tau-expressing or control cells, indicating that PHP-tau directly triggers the apoptosis machinery in the cells (Fig. $5 B$ ). In agreement, cleavage of an intracellular caspase-3 substrate, poly(ADP-ribose) polymerase (Smith, 2001), was increased in cells expressing PHP-tau compared with control cells as judged by immunoblot analysis of cellular lysates (data not shown). To test whether PHP-tau may also sensitize the cells for other apoptotic stimuli, the effects of colchicine and staurosporine, which are known to induce apoptosis in different cell types (Lindenboim et al., 1995; Ivins et al., 1999), were tested in similar survival assays. Both drugs led to a decreased survival compared with drug-free control cultures (Fig. $5 C$ ). The decrease in the relative survival was significantly higher for PHP-tau-expressing cells compared with wt-tau-expressing or control cells. Taken together, the data suggest that the expression of PHF-like tau protein has two consequences for a cell: (1) it triggers apoptosis by activating the caspase cascade, and (2) it increases the susceptibility to other apoptotic stimuli.

\section{Virus-mediated expression of pseudophosphorylated tau constructs induces neuronal degeneration in terminally differentiated human CNS model neurons}

To test the effect of tau expression in a model of terminally differentiated and polar human CNS neurons, NT2-N neurons that express fetal tau isoforms were used (Pleasure et al., 1992; Piontek et al., 1999). Because these neurons are truly postmitotic, they cannot be efficiently transfected by standard methods used for gene transfer in cell lines. Therefore, neurons were infected using a viral amplicon-based HSV-1 expression system with a panel of constructs coding for human fetal wt-tau, PHP-tau, and partially pseudophosphorylated tau constructs, where only residues in the proline-rich region $\left(\mathrm{PP}_{(\mathrm{P})}\right.$-tau) or the $\mathrm{C}$-terminal tail domain $\left(\mathrm{PP}_{(\mathrm{C})}\right.$-tau) were mutated (Fig. 6A). In addition, a construct was prepared in which all 10 residues were mutated to alanine as a nonphosphorylatable tau protein control (Ala-tau). All constructs contained a FLAG-epitope tag fused to the N terminus of the tau sequence to distinguish them from endogenous tau. Tau was expressed to a high level in infected neurons as judged by immunoblot and immunofluorescence analysis (Fig. $6 B, C)$. Depending on the number of infectious particles used for the experiments, $\leq 100 \%$ of NT2-N neurons expressed exogenous tau protein. To quantify the amount of expressed protein, the infections were adjusted to yield a ratio of $\sim 50 \%$ of tauexpressing neurons, and cellular lysates were analyzed by immunoblot relative to a recombinant tau standard. All constructs were expressed to a similar extent, which corresponded to $\sim 10^{9}$ tau molecules present per infected cell (Fig. 6B). The constructs separated at different apparent molecular weights, indicating structural changes that were induced by the mutations and phosphorylation events in the cell as described previously (Eidenmüller et al., 2000, 2001). The differences in mobility were abolished when SDS-gel electrophoresis was performed in the presence of urea (data not shown), indicating that they were caused by detergent-resistant conformational domains rather than by charge differences (Brandt et al., 1994). At the cell number analyzed, no endogenous tau was detected by immunoblot analysis because of the high level of exogenous compared with endogenous tau expression.

HSV-1-mediated gene transfer in NT2-N neurons resulted in a transient expression of tau protein, which peaked after $1 \mathrm{~d}$ and could be detected $\leq 7 \mathrm{~d}$ after infection (Fath et al., 2000). To determine whether pseudophosporylated tau exerted cytotoxicity also in this model of terminally differentiated human neurons, NT2-N neurons were infected with the different constructs and analyzed 3-4 d after infection for signs of degeneration. In this condition, $\sim 10 \%$ of wt-tau-expressing neurons showed condensed or fragmented nuclei as judged by nuclear staining with DAPI (Fig. $6 C, D$ ). Very similar numbers were obtained for Alatau-expressing cells. In contrast, approximately three times as many PHP-tau-expressing neurons exhibited a change in nuclear morphology. The increase was highly significant, indicating that PHP-tau exerts a neurotoxic effect also in NT2-N neurons. The partially mutated constructs $\left(\mathrm{PP}_{(\mathrm{P})}\right.$ - and $\mathrm{PP}_{(\mathrm{C})}$-tau) showed intermediate ratios of neurons with signs of degeneration. To test whether the cytotoxicity of pseudophosphorylated tau associated with an induction of apoptotic mechanisms, TUNEL assays were performed. Similar to the results obtained for the analysis of changes in nuclear morphology, PHP-tau-expressing neurons exhibited an increase in the percentage of TUNEL-positive cells compared with wt-tau- or Ala-tau-expressing neurons (Fig. 6E). 
A

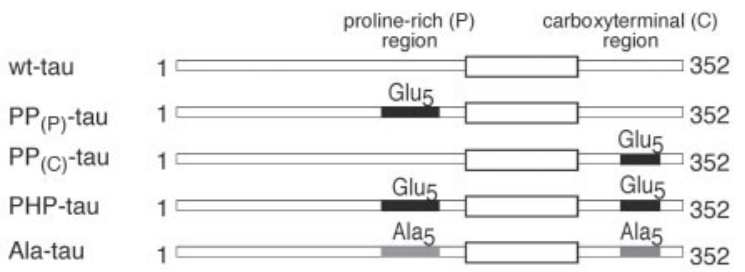

C

wt-tau

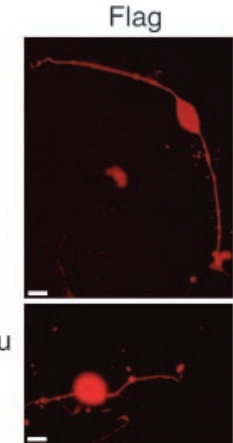

DAPI

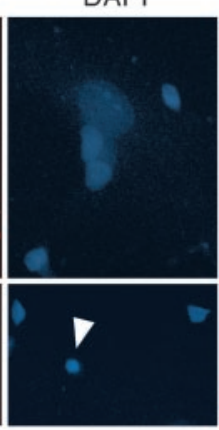

overlay

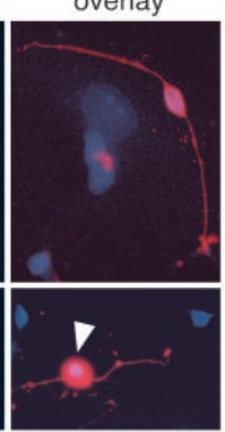

B

tau

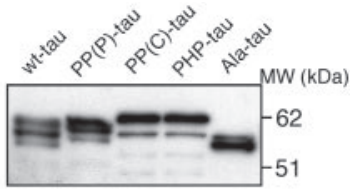

actin

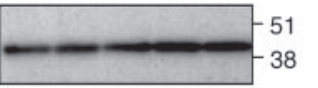

D

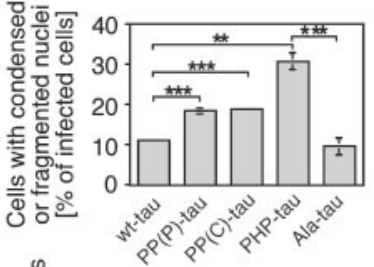

E

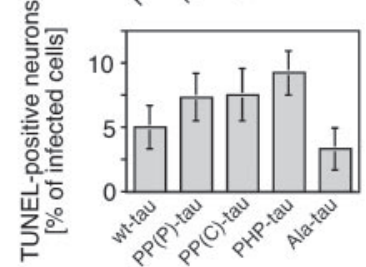

Figure 6. Virus-mediated overexpression of fetal PHP-tau and partially pseudophosphorylated constructs $\left(\mathrm{PP}_{(P)}\right.$-tau and $\mathrm{PP}_{(C)}$-tau) in human model neurons induce neuronal degeneration. $A$, Schematic of the different tau constructs generated by site-directed mutagenesis. Clusters of five serine/ threonine residues in the proline-rich $(P)$ region or the C-terminal $(C)$ region were changed to glutamate or alanine as indicated by $G l u_{5}$ or Ala $_{5}$, respectively. $B$, Immunoblot of lysates from infected model neurons expressing the indicated constructs. All constructs are expressed to a comparable level in the cell. As a control for equal loading, an actin blot is shown. Some of the constructs are present as different phosphoisoforms, as indicated by the presence of multiple bands with different electrophoretic mobilities. $C$, Fluorescence staining for exogenous tau (red) and nuclei (blue) of NT2-N neurons infected with a HSV[wt-tau] (top) or HSV[PHP-tau] (bottom) construct. Condensed nuclei in infected NT2-N neurons are indicated by arrowheads. $D, E$, Quantification of the percentage of condensed or fragmented nuclei $(D)$ and TUNEL-positive cells $(E)$ after infection with the indicated constructs. Cells infected with PHP-tau show an increased fraction of degenerated and TUNEL-positive neurons compared with wt-tau- or Ala-tau-expressing cells. The partially pseudophosphorylated constructs induce intermediate toxicity. For immunoblot analysis, 15,000 cells per well were plated on Matrigel-coated four-well plates, infected $3 \mathrm{~d}$ later, and lysed $1 \mathrm{~d}$ after infection. Lysates corresponding to 750 cells were separated by SDS-PAGE on $10 \%$ acrylamide and detected with anti-tau (Tau5) and anti-actin antibody. For immunocytochemistry, cells were plated at $2500-5000$ cells per squared centimeter on Matrigel-coated coverslips, infected 3 d later with the respective HSV-1 construct, fixed with 4\% paraformadehyde 3-4 d later, and stained against the Flag-epitope tag (M5) and with DAPI as described in Materials and Methods. D, The percentage of infected cells with condensed or fragmented nuclei was determined by visual inspection. Between 476 and 3478 infected neurons from three sets of experiments were evaluated per construct. Experiments with independently prepared virus stocks gave very similar results. $E$, The ratio of TUNEL-positive/total cells was determined by visual inspection of infected cells on four coverslips from two independent experiments. Mean and SE are shown. Scale bars, $10 \mu \mathrm{m}$.

Again, the partially mutated constructs exhibited an intermediate effect.

In postnatal brain, longer isoforms of tau protein containing up to three additional exons are also expressed (Goedert et al., 1991). To test whether pseudohyperphosphorylation of longer tau isoforms also exerts a cytotoxicity, constructs that corresponded to the fetal constructs were produced from the longest low molecular weight human tau isoform containing 441 residues (Fig. $7 A$ ), expressed in NT2-N neurons, and analyzed for changes in nuclear morphology as described above. As an additional control for tau protein toxicity, a vector expressing lacZ as an unrelated protein was prepared. Many neurons expressing PHP-tau(441) showed condensed or fragmented nuclei, whereas most wttau(441)-expressing neurons exhibited an unchanged nuclear morphology. Quantification revealed that more than twice as many PHP-tau(441)-expressing neurons showed signs of degeneration compared with wt-tau(441) or lacZ-expressing neurons (Fig. $7 B)$.

Taken together, the results indicate that pseudophosphorylation of tau proteins induces cytotoxicity in human model neurons that is closely associated with an induction of apoptotic mechanisms. Furthermore, the data provide evidence that the gain of cytotoxicity of modified tau proteins corresponds to the degree of the structural change that is induced by mutations or phosphorylation.

\section{DISCUSSION}

We have shown previously that mutated tau proteins that were designed to mimic AD-like hyperphosphorylation (pseudohyperphosphorylated tau) mimic key structural and functional aspects of hyperphosphorylated tau protein (Eidenmüller et al., 2000; Maas et al., 2000), thus providing a model to analyze consequences of a disease-like state of tau protein in cells. Here, we demonstrated that, compared with wild-type tau protein, pseudohyperphosphorylated tau exhibits a reduced microtubule binding and is deficient to promote microtubule stabilization in transfected neural cells, similar to what would be expected for phosphorylated tau protein species. Furthermore, we showed that pseudohyperphosphorylated tau specifically exerts cytotoxicity in transfected neural cell lines and virus-infected human CNS model neurons. The cytotoxicity of pseudophyperphosphorylated tau was associated with the induction of apoptosis as characterized by chromatin condensation, DNA fragmentation, and activation of the caspase cascade.

Apoptosis is known as a common mechanism of neuronal death during development and disease (Mattson, 2000; Nijhawan 

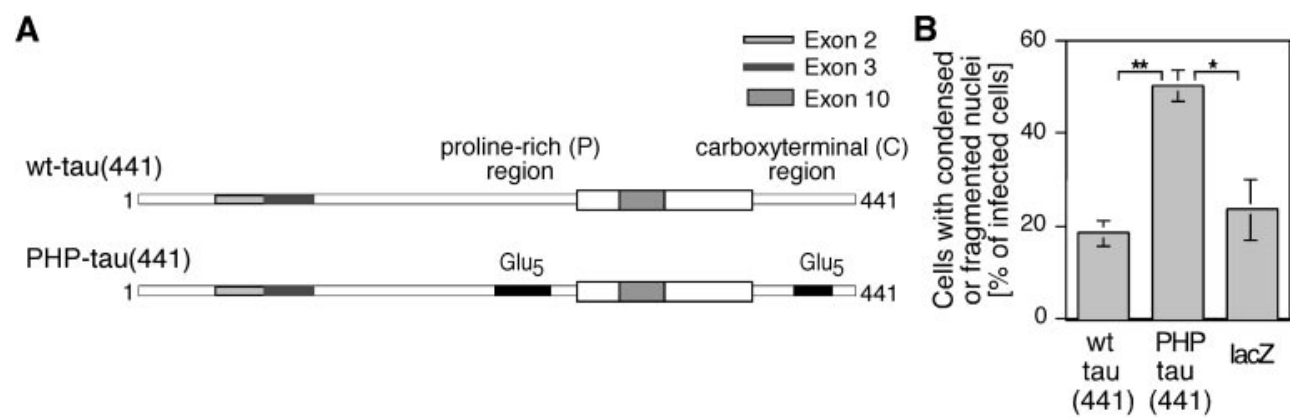

Figure 7. Virus-mediated expression of adult PHP-tau in human model neurons induces neuronal degeneration similar to the fetal constructs. $A$, Schematic of adult wt-tau and PHP-tau constructs derived from the longest low-molecular weight tau isoform. The microtubule-binding repeats of tau are indicated by the thick white box. Adult-specific exons are shaded. B, Quantification of the percentage of condensed or fragmented nuclei from neurons infected with wt-tau, PHP-tau, or, as a control, lacZ. Similar to the fetal isoforms, the longest low-molecular weight adult PHP-tau also induces neuronal degeneration. Cells were plated, infected, fixed $4 \mathrm{~d}$ after infection, and processed for immunofluorescence as described in the legend of Figure 6 . The percentage of infected cells with condensed or fragmented nuclei was determined by visual inspection. Between 86 and 203 infected neurons from four sets of experiments were evaluated per construct. Experiments with independently prepared virus stocks for wt-tau and PHP-tau gave very similar results.

et al., 2000; Yuan and Yankner, 2000). However, because of the chronic nature of $\mathrm{AD}$, the relative contribution of apoptosis is difficult to assess. Previously, postmortem analysis of human brain has shown apoptotic DNA fragmentation in AD patients ( $\mathrm{Su}$ et al., 1994; Lassmann et al., 1995; Smale et al., 1995) and elevated levels of caspase-3 activity (Gervais et al., 1999; Su et al., 2001). This suggests an important role of apoptosis during AD; however, other mechanisms may also contribute to the massive neuronal death. The proximal cause of neurodegeneration in AD remained unclear. $\mathrm{A} \beta$, the primary constituent of amyloid plaques, and the $\beta$ secretase cleavage product of amyloid precursor protein (APP) enhance apoptosis in cultured cell lines and neurons (Estus et al., 1997; Troy et al., 2000; McPhie et al., 2001), and, in turn, APP is a substrate for caspase-3, thereby increasing the concentration of $\mathrm{A} \beta$ (Gervais et al., 1999). Furthermore, mutated presenilins that cause familial Alzheimer's disease may increase neuronal vulnerability for apoptosis (Guo et al., 1997; Mattson et al., 2000; Alves da Costa et al., 2002). Previously, it has been reported that apoptotic events can result in an increased tau phosphorylation (Zhang and Johnson, 2000; Mookherjee and Johnson, 2001). Our data provide evidence that, in turn, hyperphosphorylated tau is itself capable of inducing apoptotic cell death in neurons. Because it is reported that $\mathrm{A} \beta$ can induce an increase in tau phosphorylation (Busciglio et al., 1995; Rapoport and Ferreira, 2000), hyperphosphorylation of tau may link the cytotoxic effect of $\mathrm{A} \beta$ to neurodegeneration. Interestingly, recent data show that expression of mutant APP or injection of $A \beta$ fibrils increases neurofibrillary tangle pathology of mice transgenic for tau mutations that have been found in certain tauopathies (frontotemporal dementia with Parkinsonism linked to chromosome 17) (Götz et al., 2001; Lewis et al., 2001). In addition, comparison of the effect of $A \beta$ fibrils on hippocampal neurons prepared from wild-type, tau knock-out, and human tau transgenic mice provided evidence that tau is essential for $\mathrm{A} \beta$-induced neurotoxicity (Rapoport et al., 2002). This provides additional evidence for a functional link between the effects of $\mathrm{A} \beta$, tau, and neurodegeneration.

Using ultrastructural analysis, we obtained no evidence for tau aggregation in degenerating cells that express pseudohyperphosphorylated tau protein, indicating that soluble pseudohyperphosphorylated tau without the need for previous aggregation is capable of inducing neurodegeneration. In fact, most evidence indicates that phosphorylation of tau is neutral toward or even inhibits tau aggegration in vitro (Goedert et al., 1996; Schneider et al., 1999; Eidenmüller et al., 2000), which is opposite to what would be expected if tau aggregation would have a primary role in mediating neuronal death during AD. In addition, it appears unlikely that deficits in the microtubule-related activities of tau have a primary role during neurodegeneration because PHP-tau did not actively destabilize cellular microtubules (Fig. 2D), and treatment with the microtubule-stabilizing drug taxol did not reverse the increased death of PHP-tau-expressing PC12 cells (data not shown). Thus, the data suggest that pseudohyperphosphorylated tau protein gains a toxic function in neurons rather than mediates cell death via a simple "loss of function" mechanism. In this scenario, tau aggregation appears to be a side effect rather than being causally involved in the neurodegenerative process. But what causes tau to become toxic? One may speculate that a conformational change in tau that is induced by (pseudo)hyperphosphorylation converts tau to a neurotoxic agent. In fact, pseudohyperphosphorylated tau protein shows a reduced electrophoretic mobility in SDS-PAGE, indicating the presence of detergent-resistant conformational domains that have also been observed for hyperphosphorylated tau protein (Grundke et al., 1986; Eidenmüller et al., 2000). Because partially pseudophosphorylated tau constructs induce an intermediate toxicity, this may indicate that the extent of neurotoxicity closely correlates with the degree of the structural change that is induced in tau protein. We have shown previously that modifications in the C-terminal domain induce structural changes in tau but are neutral with respect to the activity of tau on microtubules (Eidenmüller et al., 2001). Because the same construct induces neurodegeneration in a cellular context, this provides evidence that structural changes rather than functional deficiencies cause the neurons to degenerate. This is in agreement with the finding that familial mutations in tau from frontotemporal dementia-17 cases, which are also characterized by the formation of tau aggregates and death of neurons, induce structural changes in tau protein (Jicha et al., 1999a).

It is unclear which kinases and phosphatases are involved in phosphorylating tau to the degree observed in the disease. From the distribution of phosphates in hyperphosphorylated tau, it appears likely that hyperphosphorylation results from the concerted action of many kinases and phosphatases rather than from 
the activity of any individual kinase, which produces a more permanent, high stoichiometric phosphate incorporation that is simulated by the introduction of glutamate at selected sites. Our data using pseudophosphorylated constructs suggest that the cytotoxicity of tau results from structural changes that are caused by a cumulative effect of phosphate incorporation at several sites rather than from a single "hot spot" that, when phosphorylated, transforms tau into a toxic agent. This contrasts with the regulation of the interaction of tau with microtubules, which can be drastically influenced by the phosphorylation of single sites (Biernat et al., 1993; Drewes et al., 1995; Schneider et al., 1999).

Taken together, our results indicate that apoptotic mechanisms may play a role not only in the end stage of AD but also in the presymptomatic progression of the disease, when tau is still soluble but phosphorylation at PHF sites can already be detected. Second, therapeutic interventions to prevent the gain of toxic function of hyperphosphorylated tau regardless of tau aggregation may provide a useful means to slow down the progression of AD. To further clarify the intracellular mechanisms involved in hyperphosphorylation-mediated neuronal death and to identify potential drugs that interfere with this process, MTT-based cell survival assays of PHP-tau-transfected PC12 cells promise to be of help in designing high-throughput screening strategies.

\section{REFERENCES}

Alonso AC, Zaidi T, Grundke-Iqbal I, Iqbal K (1994) Role of abnormally phosphorylated tau in the breakdown of microtubules in Alzheimer disease. Proc Natl Acad Sci USA 91:5562-5566.

Alves da Costa C, Paitel E, Mattson MP, Amson R, Telerman A, Ancolio K, Checler F (2002) Wild-type and mutated presenilins 2 trigger p53dependent apoptosis and down-regulate presenilin 1 expression in HEK293 human cells and in murine neurons. Proc Natl Acad Sci USA 99:4043-4048.

Biernat J, Gustke N, Drewes G, Mandelkow E-M, Mandelkow E (1993) Phosphorylation of $\operatorname{Ser}^{262}$ strongly reduces binding of tau to microtubules: distinction between PHF-like immunoreactivity and microtubule binding. Neuron 11:153-163.

Braak E, Braak H, Mandelkow EM (1994) A sequence of cytoskeleton changes related to the formation of neurofibrillary tangles and neuropil threads. Acta Neuropathol Berl 87:554-567.

Brandt R, Lee G, Teplow DB, Shalloway D, Abdel-Ghany M (1994) Differential effect of phosphorylation and substrate modulation on tau's ability to promote microtubule growth and nucleation. J Biol Chem 269:11776-11782.

Brandt R, Léger J, Lee G (1995) Interaction of tau with the neural plasma membrane mediated by tau's amino-terminal projection domain. J Cell Biol 131:1327-1340.

Brewer GJ, Torricelli JR, Evege EK, Price PJ (1993) Optimized survival of hippocampal neurons in B27-supplemented Neurobasal, a new serum-free medium combination. J Neurosci Res 35:567-576.

Busciglio J, Lorenzo A, Yeh J, Yankner BA (1995) Beta-amyloid fibrils induce tau phosphorylation and loss of microtubule binding. Neuron 14:879-888.

Drewes G, Trinczek B, Illenberger S, Biernat J, Schmitt-Ulms G, Meyer HE, Mandelkow EM, Mandelkow E (1995) Microtubule-associated protein/microtubule affinity-regulating kinase (p110mark). A novel protein kinase that regulates tau-microtubule interactions and dynamic instability by phosphorylation at the Alzheimer-specific site serine 262 . J Biol Chem 270:7679-7688.

Eidenmüller J, Fath T, Hellwig A, Reed J, Sontag E, Brandt R (2000) Structural and functional implications of tau hyperphosphorylation: information from phosphorylation-mimicking mutated tau proteins. Biochemistry 39:13166-13175.

Eidenmüller J, Fath T, Maas T, Pool M, Sontag E, Brandt R (2001) Phosphorylation-mimicking glutamate clusters in the proline-rich region are sufficient to simulate the functional deficiencies of hyperphosphorylated tau protein. Biochem J 357:759-767.

Estus S, Tucker HM, van Rooyen C, Wright S, Brigham EF, Wogulis M, Rydel RE (1997) Aggregated amyloid-beta protein induces cortical neuronal apoptosis and concomitant "apoptotic" pattern of gene induction. J Neurosci 17:7736-7745.

Fath T, Eidenmüller J, Maas T, Brandt R (2000) Herpes simplex virusmediated expression of the axonal protein tau in human model neurons (NT2-N cells). Microsc Res Tech 48:85-96.

Gervais F, Xu D, Robertson GS, Vaillancourt J, Zhu Y, Huang J,
LeBlanc A, Smith D, Rigby M, Shearman MS, Clarke EE, Zheng H, Van Der Ploeg LHT, Ruffolo SC, Thornberry NA, Xanthoudakis S, Zamboni RJ, Roy S, Nicholson DW (1999) Involvement of caspases in proteolytic cleavage of Alzheimer's amyloid- $\beta$ precursor protein and amyloidogenic $\mathrm{A} \beta$ peptide formation. Cell 97:395-406.

Goedert M, Crowther RA, Garner CC (1991) Molecular characterization of microtubule-associated proteins tau and MAP2. Trends Neurosci 14:193-199.

Goedert M, Jakes R, Spillantini MG, Hasegawa M, Smith MJ, Crowther RA (1996) Assembly of microtubule-associated protein tau into Alzheimer-like filaments induced by sulphated glycosaminoglycans. Nature 383:550-553.

Götz J, Chen F, van Dorpe J, Nitsch RM (2001) Formation of neurofibrillary tangles in P301I tau transgenic mice induced by Abeta 42 fibrils. Science 293:1491-1495.

Greene LA, Sobeih MM, Teng KK (1991) Methodologies for the culture and experimental use of the PC12 rat pheochromocytoma cell line. In: Culturing nerve cells (Banker G, Goslin K, eds), pp 207-226. Cambridge, MA: MIT.

Grundke II, Iqbal K, Tung YC, Quinlan M, Wisniewski HM, Binder LI (1986) Abnormal phosphorylation of the microtubule-associated protein tau in Alzheimer cytoskeletal pathology. Proc Natl Acad Sci USA 83:4913-4917.

Guo Q, Sopher BL, Furukawa K, Pham DG, Robinson N, Martin GM, Mattson MP (1997) Alzheimer's presenilin mutation sensitizes neural cells to apoptosis induced by trophic factor withdrawal and amyloid beta-peptide: involvement of calcium and oxyradicals. J Neurosci 17:4212-4222.

Ivins KJ, Ivins JK, Sharp JP, Cotman CW (1999) Multiple pathways of apoptosis in PC12 cells. J Biol Chem 274:2107-2112.

Jicha GA, Rockwood JM, Berenfeld B, Hutton M, Davies P (1999a) Altered conformation of recombinant frontotemporal dementia-17 mutant tau proteins. Neurosci Lett 260:153-156.

Jicha GA, Weaver C, Lane E, Vianna C, Kress Y, Rockwood J, Davies P (1999b) cAMP-dependent protein kinase phosphorylations on tau in Alzheimer's disease. J Neurosci 19:7486-7494.

Kilmartin JV, Wright B, Milstein C (1982) Rat monoclonal antibodies derived by using a new nonsecreting rat cell line. J Cell Biol 93:576-582.

Lassmann H, Bancher C, Breitschof H, Wegiel J, Bobinski M, Jellinger K, Wiesniewski HM (1995) Cell death in Alzheimer's disease evaluated by DNA fragmentation in situ. Acta Neuropathol 89:35-41.

Lee VM, Goedert M, Trojanowski JQ (2001) Neurodegenerative tauopathies. Annu Rev Neurosci 24:1121-1159.

Lewis J, Dickson DW, Lin WL, Chisholm L, Corral A, Jones G, Yen SH, Sahara N, Skipper L, Yager D, Eckman C, Hardy J, Hutton M, McGowan E (2001) Enhanced neurofibrillary degeneration in transgenic mice expressing mutant tau and APP. Science 293:1487-1491.

Lindenboim L, Haviv R, Stein R (1995) Inhibition of drug-induced apoptosis by survival factors in PC12 cells. J Neurochem 64:1054-1063.

Lu Q, Wood JG (1993) Functional studies of Alzheimer's disease tau proteins. J Neurosci 13:508-515.

Maas T, Eidenmüller J, Brandt R (2000) Interaction of tau with the neural membrane cortex is regulated by phosphorylation at sites that are modified in paired helical filaments. J Biol Chem 275:15733-15740.

Matsuo ES, Shin R, Billingsley ML, deVoorde AV, O'Connor M, Trojanowski JQ, Lee VM (1994) Biopsy-derived adult human brain tau is phosphorylated at many of the same sites as Alzheimer's disease paired helical filament tau. Neuron 13:989-1002.

Mattson MP (2000) Apoptosis in neurodegenerative disorders. Nat Rev Mol Cell Biol 1:120-129.

Mattson MP, Zhu H, Yu J, Kindy MS (2000) Presenilin-1 mutation increases neuronal vulnerability to focal ischemia in vivo and to hypoxia and glucose deprivation in cell culture: involvement of perturbed calcium homeostasis. J Neurosci 20:1358-1364.

McPhie DL, Golde T, Eckman CB, Yager D, Brant JB, Neve RL (2001) beta-Secretase cleavage of the amyloid precursor protein mediates neuronal apoptosis caused by familial Alzheimer's disease mutations. Brain Res Mol Brain Res 97:103-113.

Mookherjee P, Johnson GV (2001) Tau phosphorylation during apoptosis of human SH-SY5Y neuroblastoma cells. Brain Res 921:31-43.

Morishima-Kawashima M, Hasegawa M, Takio K, Suzuki M, Yoshida H, Titani K, Ihara Y (1995) Proline-directed and non-proline-directed phosphorylation of PHF-tau. J Biol Chem 270:823-829.

Nijhawan D, Honarpour N, Wang X (2000) Apoptosis in neural development and disease. Annu Rev Neurosci 23:73-87.

Patrick GN, Zukerberg L, Nicolic M, de la Monte S, Dikkes P, Tsai LH (1999) Conversion of p35 tp p25 deregulates Cdk5 activity and promotes neurodegeneration. Nature 402:615-622.

Piontek J, Chen CC, Kempf M, Brandt R (1999) Neurotrophins differentially regulate the survival and morphological complexity of human CNS model neurons. J Neurochem 73:139-146. 
Piperno G, LeDizet M, Chang XJ (1987) Microtubules containing acetylated alpha-tubulin in mammalian cells in culture. J Cell Biol 104:289-302.

Pleasure SJ, Page C, Lee VMY (1992) Pure, postmitotic, polarized human neurons derived from NTera 2 cells provide a system for expressing exogenous proteins in terminally differentiated neurons. J Neurosci 12:1802-1815.

Rapoport M, Ferreira A (2000) PD98059 prevents neurite degeneration induced by fibrillar beta-amyloid in mature hippocampal neurons. J Neurochem 74:125-133.

Rapoport M, Dawson HN, Binder LI, Vitek MP, Ferreira A (2002) Tau is essential to beta-amyloid-induced neurotoxicity. Proc Natl Acad Sci USA 99:6364-6369.

Schneider A, Biernat J, von Bergen M, Mandelkow E, Mandelkow EM (1999) Phosphorylation that detaches tau protein from microtubules (Ser262, Ser214) also protects it against aggregation into Alzheimer paired helical filaments. Biochemistry 38:3549-3558.

Smale G, Nichols NR, Brady DR, Finch CE, Horton WEJ (1995) Evidence for apoptotic cell death in Alzheimer's disease. Exp Neurol $133: 225-230$

Smith CJ, Anderton BH, Davis DR, Gallo JM (1995) Tau isoform expression and phosphorylation state during differentiation of cultured neuronal cells. FEBS Lett 375:243-248.

Smith S (2001) The world according to PARP. Trends Biochem Sci 26:174-179.

Su JH, Anderson AJ, Cummings BJ, Cotman CW (1994) Immunohistochemical evidence for apoptosis in Alzheimer's disease. NeuroReport 5:2529-2533.

Su JH, Zhao M, Anderson AJ, Srinivasan A, Cotman CW (2001) Activated caspase-3 expression in Alzheimer's and aged control brain: correlation with Alzheimer pathology. Brain Res 898:350-357.

Thornberry NA, Lazebnik Y (1998) Caspases: enemies within. Science 281:1312-1316.

Troy CM, Rabacchi SA, Friedman WJ, Frappier TF, Brown K, Shelanski ML (2000) Caspase-2 mediated neuronal cell death induced by $\beta$-amyloid. J Neurosci 20:1386-1392.

Yuan J, Yankner BA (2000) Apoptosis in the nervous system. Nature 407:802-809.

Zhang J, Johnson GV (2000) Tau protein is hyperphosphorylated in a site-specific manner in apoptotic neuronal PC12 cells. J Neurochem $75: 2346-2357$. 